\title{
Aseptic cultivation of Coprinus comatus (O. F. Mull.) Gray on various pulp and paper wastes
}

\author{
Dulay $\mathrm{RMR}^{1}{ }^{*}$, Parungao AG IV ${ }^{1}$, Kalaw $\mathrm{SP}^{1,2}$ and Reyes $\mathrm{RG}^{1,2}$ \\ ${ }^{1}$ Center for Tropical Mushroom Research and Development, Central Luzon State University, Science City of Muñoz, \\ Nueva Ecija, Philippines \\ ${ }^{2}$ Department of Biological Sciences, College of Arts and Sciences, Central Luzon State University, Science City of \\ Muñoz, Nueva Ecija, Philippines
}

Dulay RMR, Parungao AG IV, Kalaw SP, Reyes RG 2012 - Aseptic cultivation of Coprinus comatus (O. F. Mull.) Gray on various pulp and paper wastes. Mycosphere 3(3), 392-397, Doi 10.5943 /mycosphere/3/3/10

The chemical components of pulp and paper wastes from industrial paper mill were analyzed prior to aseptic cultivation. Mycelial running, primordium initiation, fruiting body development, yield and bioefficiency of Coprinus comatus on pulp and paper waste supplemented with rice bran were studied. Chemical analysis revealed that brown paper waste contains $48 \mathrm{ppm}$ of $\mathrm{Pb}$. The fastest mycelia colonization ( 8 days), primordium initiation (12 days) and fruiting body development (14 days) were realized in substrate composed of pure coarse gray paper waste. However, the highest yield $(9.53 \mathrm{~g})$ and biological efficiency $(23.96 \%)$ were recorded in the formulation containing light blue paper waste $+10 \%$ rice bran. The fruiting bodies produced in contaminated paper wastes were detected to consist of $16.15 \mathrm{ppm}$ of $\mathrm{Pb}$. In general, we have successfully demonstrated the cultivation of $C$. comatus on pulp and paper wastes enriched with rice bran and its ability to absorb $\mathrm{Pb}$ from contaminated substrates.

Key words - biological efficiency - cultivation phases - heavy metals - mycoremediation

Article Information

Received 1 June 2012

Accepted 19 June 2012

Published online 30 June 2012

*Corresponding author: Rich Milton R. Dulay - e-mail - richmiltondulay@yahoo.com

\section{Introduction}

Coprinus comatus (O. F. Mull.) Gray, which is known worldwide as shaggy ink cap, lawyer's wig, and shaggy mane and locally known in the Philippines as kabuteng hapon and kabuteng demonyo due to its inky black basidiocarps (Reyes et al., 2009)-, is a member of Agaricales family, and frequently inhabit lawns, gardens and piles of rice straw. Its basidiocarps have autolytic characteristics which digest upwards from the bottom of the pileus, eventually becoming inky to liberate its basidiospores. The Volvariella growers in the country particularly in Luzon considered it as a weed fungus due to its aggressive behaviour of growing and dominating the substrates intended for $V$. volvacea cultivation, as a result of limitation in space and scarcity of nutrients (Reyes et al., 2009). Being a weed fungus in mushroom cultivation but due to its desirable nutraceutical profile, Reyes et al (2009) successfully shifted its ecological niche from being a weed fungus to an economic crop. They initiated the aseptic cultivation of $C$. comatus on sawdust-rice grit (8 parts: 2 parts $\mathrm{v} / \mathrm{v}$ ) based formulation in a miniaturized bottle under laboratory conditions. This agaric mushroom is a delicious and nutritious food source (Luo et al., 1991). It contains 8 essential amino acids namely valine, leucine, lysine, isoleucine, threonine, phenylalanine, tryptophan and methionine. The presence of $\gamma$ - 
amino butyric acid (GABA) and ornithine, the ability to inhibit the angiotensin converting enzyme confirmed its importance as a natural source of nutraceutical (Reyes et al., 2009). Additionally, it can topple blood sugar and fat, inhibit growth of tumors (Liu and Zhang, 2003) and has an antioxidative potential (Popovic et al., 2010). Although this mushroom is popular as culinary ingredient, its availability in the local market becomes limited due to its seasonal occurrence in the wild. Local mushroom hunters collect them from decomposing piles of rice straw. Cultivation of C. comatus was reported on different agricultural residues such as rice straw, cotton waste, corn cobs, urea, manure, lime (Yang and Xue, 2000), chicken manure supplemented by solid sisal waste (Lyantagaye et al., 2010). However, no attempts have been made on the cultivation on pulp and paper waste as its basal substrate especially under aseptic condition.

In the Philippines, one paper mill alone generates 100-120 tons of paper sludge per day (Fernandez et al. 2001), thus, creating major problem of waste disposal. This material mainly consists of cellulose fibre and inorganic materials. Being cellulosic, cultivation of edible mushrooms on pulp and paper waste represents one of the most efficient biological ways to convert this industrial residue into additional profit. Baysal et al (2003) successfully demonstrated the utilization of pulp and paper waste as the base material supplemented with rice husk for the yield improvement of oyster mushroom (Pleurotus ostreatus).

In the present study, we developed new production technology for $C$. comatus using pulp and paper waste as a basal medium enriched with rice bran which served as a strong foundation for industrial waste utilization.

\section{Methods}

\section{Source of strain and revival of culture}

A pure culture of $C$. comatus was obtained from the Center for Tropical Mushroom Research and Development. Agar blocks of approximately $10 \mathrm{~mm}^{2}$ x $3 \mathrm{~mm}$ from the pure stock culture of $C$. comatus were aseptically transferred into sterilized potato sucrose gulaman (PSG) plate. Culture plates were incubated at room temperature to allow growth of the secondary mycelia.

\section{Fruiting body performance of $C$. comatus on paper pulp formulations}

The production of fruiting bodies of $C$. comatus was evaluated on pulp and paper waste from an industrial paper mill as a base substrate. Initially, four samples of pulp and paper wastes (fine gray, coarse gray, light blue and brown) were brought to the Analytical Services Laboratory of the Philippine Rice Research Institute for chemical characterization of the pulp and paper waste ingredients. The protocol of Reyes et al (2009) on aseptic cultivation was adopted with minor modifications. Pulp and paper wastes were moistened to gain 65\% moisture content (MC). Following the formulations (pure pulp and paper waste and an addition of rice bran as supplement) of the different pulp and paper wastes, 40 grams of each formulated material was placed in a miniaturized glass bottle occupying approximately half to give space for the appearance of fruiting bodies. Bottled substrates were individually covered with polypropylene sheets, secured with a rubber band and sterilized at $15 \mathrm{psi}, 121{ }^{\circ} \mathrm{C}$ for 45 minutes. Each was inoculated with a $10 \mathrm{~mm}$ diameter mycelia disc of the revived culture of $C$. comatus and subsequently incubated at room temperature $30 \pm 2{ }^{\circ} \mathrm{C}$ under alternating light and dark condition. After mycelial colonization, the bottled cultures were placed into a growing chamber with $80-90 \% \mathrm{RH}$ to allow the primordium initiation and fruiting body development.

Each treatment was replicated 3 times in 2 experimentation set-ups. The number of days was recorded after the total mycelial colonization of the substrate, primordium initiation and fruiting body development were observed. The weight of non-mature fruiting bodies was determined and percentage biological efficiency was computed. Data were analyzed using analysis of variance (ANOVA) in one way classification analysis. Duncan Multiple Range Test (DMRT) was used to determine the significant treatment comparison at $5 \%$ level of significance. The Sirichai Statistics 6.07 computer program was used for analysis. 


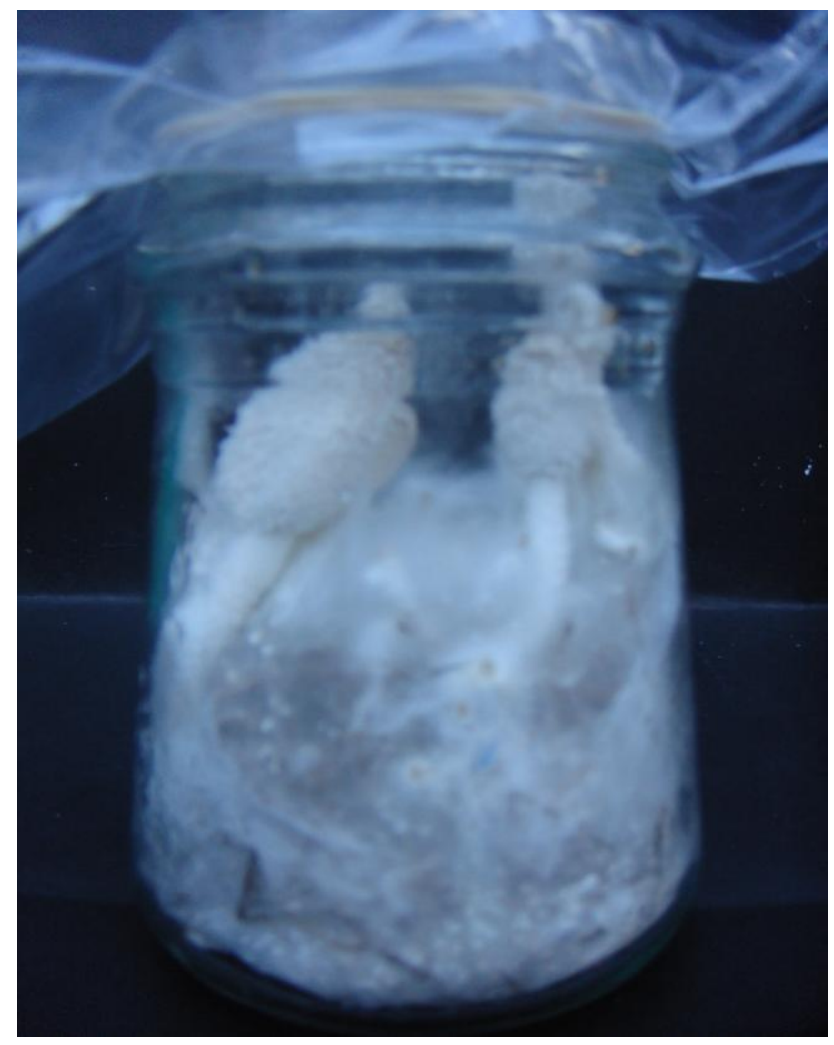

Fig. 1 - Fruiting bodies of $C$. comatus grown on LBPP + RB medium in a miniaturized glass bottle.

\section{Results and Discussion}

\section{Chemical contents of pulp and paper wastes}

A leading industrial paper mill in the country that generates a huge pulp and paper sludge daily provides pulp and paper wastes. Disposal of this waste is becoming a major problem for the industry. We intervened to create efficient means of its utilization, i.e. through mushroom cultivation. But prior to that, the four types of pulp and paper waste were analyzed to determine the chemical contents and to check their safety to humans. The organic carbon, nitrogen, $\mathrm{pH}$ and lead $(\mathrm{Pb})$ content of the different pulp and paper wastes are presented in Table 1. Among the samples, fine and coarse gray pulp and paper waste had the highest organic carbon and the lowest amount of nitrogen. Light blue pulp and paper waste had the lowest organic carbon but higher amount of nitrogen when compared with the nitrogen content of the fine and coarse gray pulp and paper waste. In terms of $\mathrm{pH}$, all samples were basic with a $\mathrm{pH}$ range of 8-10. Though it is imperative to reformulate these substrates with nutritional supplements to adjust the $\mathrm{pH}$ condition and improve the nutrients, obviously, pulp and paper wastes hold promising nutritional value for excellent growth performance of $C$. comatus. Paper sludge is a mixture of solid chemical residues in the process of manufacturing and bleaching the pulp and paper. It is also known to contain heavy metals (Ismail et al, 2010). In this study, it is revealed that among the four samples, brown pulp and paper waste contained $48 \mathrm{ppm}$ of lead. It is therefore, not recommended as a substrate for mushroom cultivation.

\section{Mycelial colonization, primordial initiation and fruiting body development}

The time periods of the different phases of cultivation of $C$. comatus are given in Table 2. The formulation containing pure CGPP had the shortest period of mycelial colonization ( 8 days), primordium initiation (12 days), and fruiting body development (14 days). It was followed by FGPP + RB with 9.33 days, 12.67 days, 14.33 days, in respective phases of cultivation, which was statistically comparable with pure FGPP. Despite of the relatively long time periods of fructification in these formulations compared to 8 days when grown in sawdust-rice grit $(8: 2 \mathrm{v} / \mathrm{v})$ formulation, it is better than 17 days when spawned in sawdustrice bran $(8: 2 \mathrm{v} / \mathrm{v})$ formulation (Reyes et al., 
Table 1 Organic carbon, nitrogen, $\mathrm{pH}$ and lead $(\mathrm{Pb})$ content of four types of pulp and paper wastes.

\begin{tabular}{lcccc}
\hline Pure Pulp/Paper Waste & Organic Carbon (\%) & Nitrogen (\%) & pH & Lead (ppm) \\
\hline Fine gray paper pulp (FGPP) & $90.40 \pm 0.40$ & $0.14 \pm 0.01$ & $10 \pm 0$ & ND \\
Coarse gray paper pulp (CGP) & $90.00 \pm 0.20$ & $0.11 \pm 0.01$ & $9 \pm 0$ & ND \\
Light blue paper pulp (LBPP) & $32.80 \pm 0.10$ & $0.29 \pm 0.00$ & $9 \pm 0$ & ND \\
Brown paper pulp (BPP) & $52.60 \pm 0.30$ & $0.51 \pm 0.01$ & $8 \pm 0$ & 48 \\
\hline
\end{tabular}

(In Table: Values are the mean \pm SD of chemical contents of paper waste. ND: not detected, lead detection limit is 2 ppm)

2009). Findings of the present study suggest that among the pure pulp and paper wastes evaluated, CGPP is the most suitable substrate for the various phases of $C$. comatus cultivation due to high amount organic carbon and low amount of nitrogen. Though CGPP and FGPP contain almost the same amount of nutrients (C:N in Table 1), FGPP had higher $\mathrm{pH}(9.6)$, which was excessively higher with the optimum $\mathrm{pH}$ range of $6.0-8.0$ of $C$. comatus (Jang et al., 2009). This relatively high pH triggers the slow mycelial running. But addition of rice bran to FGPP stimulates cultivation, which might be attributed to the enrichment of the formulation and the adjustment of the $\mathrm{pH}$. However, pure LBPP recorded the most extensive period in all phases of cultivation most likely due to very low organic carbon and relatively high amount of nitrogen, which is known to inhibit mushroom growth in excessive amounts (Demirci, 1998). Addition of rice bran to LBPP shortens the time periods in all phases of cultivation by $33 \%$.

\section{Yield and Biological Efficiency}

After determining the most favorable substrate for the various phases of cultivation, the yield and biological efficiency of $C$. comatus cultivation were also evaluated. There were significant differences found in the mean yield within three to four flushes among the different substrate formulations (Table 2). It was revealed that addition of $10 \%$ rice bran to the pulp and paper waste substrates significantly increased the mean yield compared to pure pulp and paper waste substrates. The LBPP $+\mathrm{RB}$ formulation produced the highest mean yield of $9.58 \mathrm{~g}$ with a biological efficiency of $23.96 \%$. Moreover, the largest fruiting bodies were also observed on this substrate (Figure 1). The data gathered in this study are better than the previous report of $14 \%$ bio-efficiency when the mushroom was grown in formulated sawdust-rice bran substrates and $18 \%$ bio-efficiency in sawdustrice grit combination (Reyes et al., 2009). However, pure CGPP which provided the efficient cultivation properties recorded the lowest bio-efficiency of $0.12 \%$. Thus, addition of rice bran to pulp and paper waste as substrate for cultivation of $C$. comatus amends the chemical contents which positively affected the mushroom yield.

Since fungi are known to accumulate heavy metals (Tyler, 1980; Bano et al., 1981), we have investigated the ability of $C$. comatus to accumulate lead $(\mathrm{Pb})$, which was detected in BRPP. The dried fruiting bodies harvested on this substrate contained $16.15 \mathrm{ppm}$ of lead. This significant result indicates that this mushroom can be an agent for $\mathrm{Pb}$ mycoremediation. Also, this implies that substrates must be lead free before utilizing them for the mushroom cultivation.

Industrial pulp and paper wastes enriched with $10 \%$ rice bran can be used for the cultivation of $C$. comatus. Pure paper pulps stimulate the cultivation properties but resulted to low yield. However, addition of rice bran to pulp and paper waste significantly increased the mushroom yield and enhanced bioefficiency. The present study also revealed that C. comatus accumulates lead $(\mathrm{Pb})$ from the contaminated pulp and paper waste substrate. Furthermore, cultivation of other wild edible mushrooms on clean and safe pulp and paper waste as basal medium enriched with rice bran and other supplements is recommended. 
Table 2 Mycelial colonization, primordium initiation, fruiting body development, mushroom yield and biological efficiency of $C$. comatus.

\begin{tabular}{|c|c|c|c|c|c|}
\hline Substrate & $\begin{array}{l}\text { Mycelial } \\
\text { colonization } \\
\quad(\text { day })\end{array}$ & $\begin{array}{l}\text { Primordium } \\
\text { initiation } \\
\text { (day) }\end{array}$ & $\begin{array}{l}\text { Fruiting body } \\
\text { development } \\
\text { (day) }\end{array}$ & $\begin{array}{l}\text { Yield } \\
\text { (gram) }\end{array}$ & $\begin{array}{l}\mathbf{B E}^{\mathbf{b}} \\
(\%)\end{array}$ \\
\hline FGPP (pure) ${ }^{a}$ & $9.67 \pm 0.58^{\mathrm{d}}$ & $13.67 \pm 0.58^{\mathrm{d}}$ & $14.67 \pm 0.58^{\mathrm{d}}$ & $0.14 \pm 0.07^{\mathrm{d}}$ & 0.35 \\
\hline CGPP (pure) & $8.00 \pm 0.00^{\mathrm{e}}$ & $12.00 \pm 0.00^{\mathrm{e}}$ & $14.00 \pm 0.00^{\mathrm{d}}$ & $0.05 \pm 0.01^{\mathrm{d}}$ & 0.12 \\
\hline LBPP (pure) & $18.00 \pm 1.00^{\mathrm{a}}$ & $21.00 \pm 1.00^{\mathrm{a}}$ & $23.33 \pm 0.58^{\mathrm{a}}$ & $0.67 \pm 0.14^{\mathrm{d}}$ & 1.67 \\
\hline BRPP (pure) & $13.67 \pm 0.58^{\mathrm{b}}$ & $16.33 \pm 0.58^{\mathrm{bc}}$ & $18.67 \pm 0.58^{\mathrm{bc}}$ & $0.55 \pm 0.18^{\mathrm{d}}$ & 1.37 \\
\hline $\mathrm{FGPP}+\mathrm{RB}$ & $9.33 \pm 0.58^{\mathrm{d}}$ & $12.67 \pm 0.58^{\mathrm{de}}$ & $14.33 \pm 0.58^{\mathrm{d}}$ & $5.70 \pm 0.21^{\mathrm{b}}$ & 14.26 \\
\hline $\mathrm{CGPP}+\mathrm{RB}$ & $12.67 \pm 0.58^{\mathrm{bc}}$ & $16.67 \pm 0.58^{\mathrm{bc}}$ & $18.33 \pm 1.15^{\mathrm{c}}$ & $6.16 \pm 0.43^{b}$ & 15.40 \\
\hline $\mathrm{LBPP}+\mathrm{RB}$ & $12.00 \pm 0.00^{\mathrm{c}}$ & $15.67 \pm 1.53^{\mathrm{c}}$ & $19.33 \pm 2.89^{\mathrm{bc}}$ & $9.58 \pm 2.23^{\mathrm{a}}$ & 23.96 \\
\hline $\mathrm{BRPP}+\mathrm{RB}$ & $12.67 \pm 0.58^{\mathrm{bc}}$ & $17.33 \pm 0.58^{\mathrm{b}}$ & $20.67 \pm 0.58^{\mathrm{b}}$ & $4.25 \pm 0.22^{\mathrm{c}}$ & 10.62 \\
\hline
\end{tabular}

(In Table: Values are the mean \pm SD of time periods of cultivation phases (days) and yield (grams), and means having the same letter of superscript in the same column are insignificantly different from each other at $5 \%$ level of significance. ${ }^{a}$ FGPP: fine gray, CGPP: coarse gray, LBPP: light blue, and BRPP: brown pulp and paper waste, RB: $10 \%$ rice bran. ${ }^{\mathrm{b}}$ Biological Efficiency $(\%)=($ mean yield $/ 40 \mathrm{~g}$ wt of substrate $\left.) \mathrm{x} 100.\right)$

\section{Acknowledgements}

The authors are in debt with the staff namely Archie, Kuya Lito and Kuya Roger of the Center for Tropical Mushroom Research and Development.

\section{References}

Bano Z, Nagaraja KV, Vibhakar S, Kapur OP. 1981 - Mineral and heavy metal contents in the sporophores of Pleurotus species. Mushroom News II Letters for the Tropics 2, 3-7.

Baysal E, Peker H, Yalinkilic MK, Temiz A. 2003 - Cultivation of oyster mushroom on waste paper with some added supplementary materials. Bioresource Technology 89, 95-97.

Demirci Z. 1998 - Utilization of nonwoody and woody waste products for Pleurotus ostreatus cultivation in the region of Eastern Black Sea. M.Sc. Thesis, Trabzon, Turkey, $67 \mathrm{pp}$.

Fernandez EC, Lamason CRG, Delgado TS. 2001- Housing construction material from paper mill sludge. In: Proceedings of the 5th International Workshop on the Use of Paper Industry Sludges in Environmental Geo-Technology and Construction. pp. 37-49.

Ismail MC, Ismail MA, Lau SK, Muhammad B, Majid Z. 2010 - Fabrication of bricks from paper sludge and palm oil fuel ash.
Concrete Research Letters 1, 60-66.

Jang MJ, Lee YH, Liu JJ, Ju YC. 2009 Optimal conditions for the mycelial growth of Coprinus comatus strains. Mycobiology 37(2), 103-108.

Liu YF, Zhang JS. 2003 - Recent advances in the studies on the medicinal functions of Coprinus comatus. Acta Edulis Fungi 10(2), 60-63.

Luo XY, Lü DP, Wang W. 1991 - Artifical culture of Coprinus comatus Kunyan C901. Edible Fungi of China 10, 13-15.

Lyantagaye SL, Mwita LN, Mshandete AM. 2010 - Improved antimicrobial activity of the Tanzanian edible mushroom Coprinus cinereus (Schaeff) Gray by chicken manure supplemented solid sisal wastes substrates. Journal of Yeast and Fungal Research 1(10), 201-206.

Popović M, Vukmirović S, Stilinović N, Čapo N, Jakovljević V. 2010 - Anti-oxidative activity of an aqueous suspension of commercial preparation of the mushroom Coprinus comatus. Molecules 15, 45644571.

Reyes RG, Lopez LLM, Kumakura K, Kalaw SP, Kikukawa T, Eguchi F. 2009 Coprinus comatus, a newly domesticated wild nutraceutical mushroom in the Philippines. Journal of Agricultural Technology 5(2), 299-316.

Tyler G. 1980 - Metals in sporophores of basidiomycetes. Transactions of the 
Mycosphere Doi 10.5943/mycosphere/3/3/10

British Mycological Society 74 (1), 4149.

Yang, G.L., Xue, H.B., 2000. Specialized cultivation manual about edible and medicinal mushroom. China Agricultural Press. pp. 361-368. 